Tema: Aciaria Oxigênio

\title{
INFLUÊNCIA DAS FASES SÓLIDAS NA DESFOSFORAÇÃO DO AÇO*
}

\section{Resumo}

\author{
Sábata Marla Reis Durães Oliveira ${ }^{1}$ \\ Osíris Callegari Ferrari \\ José Roberto de Oliveira ${ }^{3}$ \\ Hugo Filipe Parreiras ${ }^{4}$ \\ Carlos André Ruy Carneiro 4 \\ Túlio Carvalho da Silva Araújo ${ }^{5}$
}

Um dos problemas enfrentados pela maior parte das aciarias relaciona-se à redução do percentual de fósforo do aço. Reduzir o fósforo contido no aço tornou-se um requisito fundamental nas siderúrgicas que utilizam convertedores LD, devido à crescente demanda do mercado por aços especiais, como por exemplo, aços para a indústria automobilística. Durante o processo de desfosforação, as principais fases sólidas que podem se formar na escória de convertedor são $\mathrm{CaO}, \mathrm{MgO}$, silicato dicálcico $\left(2 \mathrm{CaO} . \mathrm{SiO}_{2}\right)$, fosfato tricálcico $\left(3 \mathrm{CaO} . \mathrm{P}_{2} \mathrm{O}_{5}\right)$, formado pela oxidação do fósforo, e o $3 \mathrm{CaOSiO}_{2} \cdot 3 \mathrm{CaOP}_{2} \mathrm{O}_{5}$. O objetivo deste trabalho é estudar a formação destes compostos e avaliar a influência das fases sólidas formadas durante o processo de desfosforação do aço. Para isto foram analisadas quatro composições químicas de escórias com diferentes teores de $\mathrm{CaO}$ e $\mathrm{FeO}$, sendo duas composições médias das escórias estudadas pela Usiminas, uma escória da literatura e uma escória desfosforante elaborada para pesquisa. Os resultados mostraram em quais composições ocorrem a formação das fases sólidas citadas e sua influência no processo.

Palavras-chave: Convertedores LD; Desfosforação; Fases sólidas.

\section{Abstract}

INFLUENCE OF SOLID PHASES IN STEEL DEPHOSPHORIZATION

One of the problems faced by most of the steelmakings is related to the reduction in the percentage of phosphorus in steel. Reduce phosphorus contained in steel has become a key requirement in steelmakers using LD converters, due to the increasing market demand for special steels, such as steels for the automotive industry. During the process of dephosphorization, a main solid phases that can form in slag is dicalcium silicate $\left(2 \mathrm{CaO} . \mathrm{SiO}_{2}\right)$, tricalcium phosphate $\left(3 \mathrm{CaO} . \mathrm{P}_{2} \mathrm{O}_{5}\right)$, formed by the oxidation of phosphorus, and the $3 \mathrm{CaOSiO}_{2} \cdot 3 \mathrm{CaOP}_{2} \mathrm{O}_{5}$. The aim of this work is to study the formation of these compounds and to evaluate the influence of the solid phases formed during the process of dephosphorization of steel. For this four chemical compositions of slag with different $\mathrm{CaO}$ and $\mathrm{FeO}$ were analyzed, two are average compositions of slag studied by Usiminas, one literature slag and one slag elaborated for search. The results showed which compositions occur the formation of solid phases mentioned and their influence in the process.

Keywords: LD Converter; Dephosphorization; Solid Phases.

1 Mestranda, Programa de Pós-Graduação em Engenharia Metalúrgica e de Materiais, IFES, Vitória, ES, Brasil.

2 Estudante de graduação, curso de Engenharia Metalúrgica, IFES, Vitória, ES, Brasil.

3 Engenheiro Metalurgista, Professor Doutor e Coordenador, Programa de Pós-Graduação em Engenharia Metalúrgica e Materiais, IFES, Vitória, ES, Brasil.

4 Engenheiro Metalurgista, Gerência de Pesquisa e Desenvolvimento de Processos, Usiminas, Ipatinga, Minas Gerais, Brasil.

5 Engenheiro de Materiais, Gerência de Convertedores da Aciaria 2, Usiminas, Ipatinga, Minas Gerais, Brasil.

* Contribuição técnica ao 450 Seminário de Aciaria - Internacional, 25 a 28 de maio de 2014, Porto Alegre, RS, Brasil. 


\section{INTRODUÇÃO}

O processo de desfosforação do aço é realizado com a finalidade de ajustar a composição da liga metálica de acordo com parâmetros pré-estabelecidos à aplicação final do metal.

De acordo com a teoria molecular, a desfosforação pode ser descrita baseada na oxidação do fósforo e simultânea fixação do óxido resultante pelo $\mathrm{CaO}$ de acordo com a reação [1]:

$$
2 \mathrm{P}+5(\mathrm{FeO})+3(\mathrm{CaO})=\left(3 \mathrm{CaO} \cdot \mathrm{P}_{2} \mathrm{O}_{5}\right)+5 \underline{\mathrm{Fe}}
$$

A partir da reação acima, é possível obter uma relação que permite verificar alguns fatores termodinâmicos que influenciam na desfosforação, tais como temperatura, atividade da cal, potencial de oxigênio, dentre outros, conforme equação 1.

Sendo:

$$
\ln [\% P]=\frac{1}{2} \ln a_{(3 \text { CaO.P2O5) }}+\frac{\Delta G^{0}}{2 R T}-\frac{5 \mu_{O_{2}}}{4 R T}-\frac{3}{2} \ln a_{(c a O)}-\ln f_{p}
$$

- a : Atividade raoultiana dos compostos presentes na escória.

- R: Constante universal dos gases (J/mol.K).

- T: Temperatura (K).

- $\mu_{\mathrm{O}_{2}}$ : Potencial de oxigênio.

- $f_{P:}$ Coeficiente de interação do fósforo no metal.

Existem outros fatores termodinâmicos que devem ser levados em consideração no estudo da desfosforação, como por exemplo, o coeficiente de partição do fósforo e a basicidade da escória.

\subsection{Coeficiente de Atividade do $\mathrm{P}_{2} \mathrm{O}_{5}$}

A remoção do fósforo do metal e a consequente fixação do óxido formado na escória dependem da atividade do $\mathrm{P}_{2} \mathrm{O}_{5}$ na escória, a qual é função da composição química e temperatura. Turkdogan e Pearson [2] obtiveram uma relação empírica que descreve a variação do coeficiente de atividade do $\mathrm{P}_{2} \mathrm{O}_{5}$ com a temperatura e composição da escória.

$$
\log \left(\gamma_{\mathrm{P}_{2} \mathrm{O}_{5}}\right)=-1,12 \cdot\left(22 \cdot N_{\mathrm{CaO}}+15 \cdot N_{\mathrm{MgO}}+13 \cdot N_{\mathrm{MnO}}+12 \cdot N_{\mathrm{FeO}}-2 \cdot N_{\mathrm{SiO}_{2}}\right)-\frac{42000}{T}+23,58
$$

\subsection{Coeficiente de Partição do Fósforo}

O coeficiente de partição do fósforo expressa a relação de equilíbrio entre a concentração de fósforo na escória $\left(\% \mathrm{P}_{\text {eq }}\right)$ e a concentração de fósforo no metal $\left[\% \mathrm{P}_{\text {eq }}\right]$. Tal relação é mostrada na equação 3.

$$
L_{P}=\frac{\left(\% P_{e q}\right)}{\left[\% P_{e q}\right]}
$$

Balajiva et al. [3], foram os primeiros a proporem uma expressão para o coeficiente de partição do fósforo. Suito e Inoue [4] alteraram a formulação daqueles autores, conforme equação 4.

$$
\log \left\{\frac{(\% P)}{[\% P]}\right\}=0,072\{(\% \mathrm{CaO})+0,3 .(\% \mathrm{MgO})+0,6 .(\% \mathrm{MnO})+0,6 .(\% \mathrm{P} 2 \mathrm{O})\}+2,5 . \log (\% \mathrm{~T} . \mathrm{Fe})+\frac{11570}{T}-10,52
$$

Healy [5] também propôs uma expressão para o coeficiente de partição do fósforo:

\footnotetext{
* Contribuição técnica ao 450 Seminário de Aciaria - Internacional, 25 a 28 de maio de 2014, Porto Alegre, RS, Brasil.
} 


\section{ACJARIA}

$\log \frac{(\% P)}{[\% P]}=\frac{22350}{T}+0,08 .(\% C a O)+2,5 \cdot \log \left(\% F e_{\text {total }}\right)-16$

Turkdogan [1] propôs uma relação do coeficiente de partição do fósforo com o oxigênio dissolvido no banho a basicidade ótica da escória:

$\log \frac{(\% P)}{[\% P]}=\frac{21740}{T}-9,87+0,071 B O+2,5 \cdot \log (\% O)$

Sendo:

$$
\begin{gathered}
B O=(\% \mathrm{CaO})+(\% \mathrm{CaF} 2)+0,3(\% \mathrm{MgO}) \\
{[\% \mathrm{O}]=(0,1 T-155,3) \cdot 10^{\wedge}-4 .(\% \mathrm{FeO})}
\end{gathered}
$$

Mais recentemente Basu [6] propôs o cálculo da partição com base nas frações de cátions e ânions presentes na escória:

$$
\log \frac{(\% P)}{[\% P]}=1,97 \cdot X_{C a+2}+2,0 \cdot X_{F e+2}-2,04 \cdot X_{S i O 4}+4+\frac{6713}{T}-1,84
$$

\subsection{Basicidade}

O conceito de basicidade, na metalurgia, é empírico e arbitrário. A noção de ácido e base deve-se à observação de que as escórias ricas em sílica atacam refratários dolomiticos ou magnesianos e, vice-versa, escórias ricas em $\mathrm{CaO}$ e $\mathrm{MgO}$ atacam refratários siliciosos [7]. Assim, a basicidade expressa a relação, $B$, entre as concentrações de óxidos básicos e as de óxidos ácidos, ou seja:

$$
B=\frac{(\% \mathrm{CaO})}{(\% \mathrm{SiO})}
$$

A Figura 1 mostra a relação $\left[\left(\% \mathrm{P}_{2} \mathrm{O}_{5}\right) / \% \mathrm{Peq}\right]$ com a $\% \mathrm{FeO}$ na escória, correlacionando com a basicidade $\left[(\% \mathrm{CaO}) /\left(\% \mathrm{SiO}_{2}\right)\right]$.

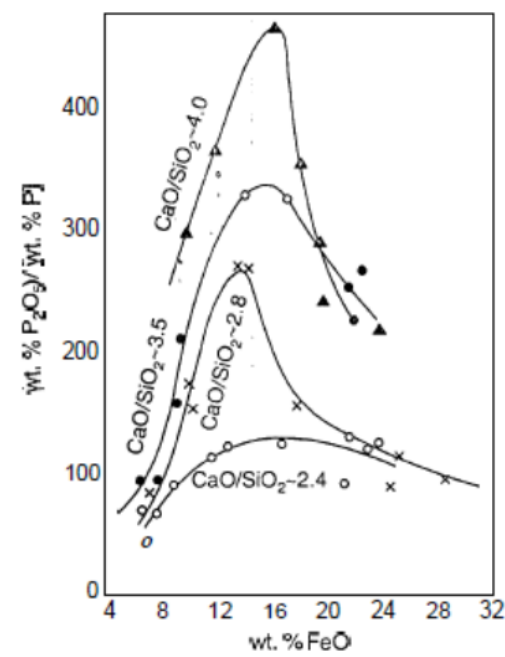

Figura 1. Dependência da relação $\left[\left(w t \% \mathrm{P}_{2} \mathrm{O}_{5}\right) / w t \% \mathrm{P}\right]$ com a $\% \mathrm{FeO}$ na escória para diferentes valores de basicidade $\left(\mathrm{CaO} / \mathrm{SiO}_{2}\right)[8]$.

Analisando-se a Figura 1 pode-se concluir que uma escória ideal para desfosforação deve apresentar basicidade 4,0 e teor de FeO em torno de $16 \%$.

* Contribuição técnica ao 450 Seminário de Aciaria - Internacional, 25 a 28 de maio de 2014, Porto Alegre, RS, Brasil. 


\subsection{Formação das Fases Sólidas}

Para analise do comportamento da escória durante o refino do aço em relação à desfosforação, é importante se conhecer as composições química e mineralógica da mesma. As principais fases presentes na escória de convertedor LD são o silicato dicálcio $\left(2 \mathrm{CaO} . \mathrm{SiO}_{2}\right)$, a magnésia-wustita ( $\left.\mathrm{MgO} . \mathrm{FeO}\right)$, e a cálcio-ferrita ( $\left.\mathrm{CaO} . \mathrm{FeO}\right)$, sendo a primeira presente em maior quantidade. De acordo com alguns autores [912], o fósforo encontra-se na forma de fosfato tricálcio e forma solução sólida com o silicato dicálcio.

Alguns autores propuseram que $\circ \mathrm{P}_{2} \mathrm{O}_{5}$ se move para a fase de silicato dicálcio, já precipitada, por meio de difusão no estado sólido durante a solidificação da escória [12]. Entretanto, é sugerido também que uma solução sólida de $3 \mathrm{CaO} \cdot \mathrm{P}_{2} \mathrm{O}_{5} \mathrm{e}$ $2 \mathrm{CaO} . \mathrm{SiO}_{2}$ precipita diretamente da escória líquida, considerando que a cinética de difusão do fósforo pela fase sólida seria muito lenta [13].

\subsection{Cinética da Desfosforação}

A desfosforação é influenciada também por fatores cinéticos, o qual se destaca o transporte de massa do fósforo a partir do seio do banho metálico até a interface metal-escória. Os modelos termodinâmicos não consideram este fenômeno, porém é importante que a cinética da reação de desfosforação seja estudada [14].

A equação que descreve a cinética de DeP passa a ser representada pela seguinte relação [15].

$$
-\frac{d[\% P]}{d t}=k\left\{\left[\% P_{i}\right]-\left[\% P_{e q}\right]\right\}
$$

\subsection{Termodinâmica Computacional}

A desfosforação envolve reações metal-escória e/ou gás-escória e determinar as condições de equilíbrio nestes sistemas é um trabalho complexo. Sendo assim, os softwares de termodinâmica computacional atuam de forma a simular as condições de equilíbrio do sistema em análise, bem como identificar as fases presentes tanto no metal quanto na escória que são funções da temperatura, pressão e composição química [16].

Os softwares de termodinâmica computacional mais utilizados são o FactSage, GEMINI2, MPE, MTDATA e o ThermoCalc, os quais apresentam muitas aplicações nas áreas química, metalúrgica e de materiais [17]. Neste trabalho o software utilizado foi o Factsage 6.2.

Este trabalho, portanto, tem como objetivo avaliar por meio da termodinâmica computacional a influência das fases sólidas na desfosforação do aço.

\section{MATERIAL E MÉTODOS}

Para a execução desse trabalho foram estudadas diferentes escórias com teores de $\mathrm{CaO}$ e $\mathrm{FeO}$ diferentes, a fim de se determinar os coeficientes de partição de acordo com os diferentes modelos propostos na literatura e as fases presentes nestas diferentes escórias. Foram estudadas as seguintes etapas:

- Escórias geradas na empresa Usiminas;

- Escória citada na literatura;

- Escória utilizada em processo siderúrgico com \%FeO acima de 30\%.

* Contribuição técnica ao 450 Seminário de Aciaria - Internacional, 25 a 28 de maio de 2014, Porto Alegre, RS, Brasil. 
Para estas escórias, como citado anteriormente, foram determinados os coeficientes de partição do fósforo de acordo com os diferentes modelos e as fases presentes nestas diferentes escórias. A determinação das fases presentes nas escórias foi feita por via instrumental e através do software de termodinâmica computacional Factsage, nas temperaturas de processo de cada um.

\subsection{Escórias Geradas na Empresa Usiminas}

A USIMINAS realizou recente pesquisa das suas escórias de maneira a tentar atingir um teor de $\mathrm{CaO}$ livre de aproximadamente $0,8 \%$ para garantir ligeira saturação da escória e o máximo teor de $\mathrm{CaO}$ dissolvido com segurança na escória final de sopro. Para isto, reduziu-se a basicidade em 0,5 unidades nas corridas cuja basicidade visada fosse igual ou superior a 4,5. Além disso, as escórias foram caracterizadas por meio da técnica de difratometria de raios-X [18].

No presente trabalho foram utilizadas as médias das escórias estudadas pela empresa antes e depois das modificações realizadas, dadas na Tabela 1. A escória chamada de EUA representa a média da composição das escórias antes das modificações e EUD, representa a média da composição das escórias depois das modificações.

Tabela 1. Composição química das médias das escórias estudadas pela Usiminas

\begin{tabular}{|c|c|c|c|c|c|c|c|c|}
\hline ESCÓRIAS & $\begin{array}{c}\mathrm{FeO} \\
(\%)\end{array}$ & $\begin{array}{c}\mathrm{CaO} \\
(\%)\end{array}$ & $\begin{array}{c}\mathrm{SiO}_{2} \\
(\%)\end{array}$ & $\begin{array}{c}\text { MnO } \\
\text { (\%) }\end{array}$ & $\begin{array}{l}\mathrm{P}_{2} \mathrm{O}_{5} \\
(\%)\end{array}$ & $\begin{array}{c}\mathrm{Al}_{2} \mathrm{O}_{3} \\
(\%)\end{array}$ & $\begin{array}{c}\mathrm{MgO} \\
\text { (\%) }\end{array}$ & $\mathrm{T}\left({ }^{\circ} \mathrm{C}\right)$ \\
\hline EUA & 24,70 & 47,20 & 11,08 & 5,74 & 2,11 & 1,71 & 9,26 & 1600 \\
\hline EUD & 24,80 & 45,30 & 11,17 & 5,74 & 2,59 & 1,71 & 9,26 & 1600 \\
\hline
\end{tabular}

\subsection{Escória Citada na Literatura (EL), e Escória com Alto Teor de FeO (EF)}

A escolha da escória citada na literatura foi feita por meio da análise da Figura 1. Foi tomada a escória que através da figura permitisse o maior valor de Lp, onde o teor de $\mathrm{FeO}$ é de $16 \%$ e a basicidade $\left(\mathrm{CaO} / \mathrm{SiO}_{2}\right)$ da escória igual a 4,0.

A escória com alto teor de $\mathrm{FeO}$ foi a escolhida com base em uma escória utlizada por empresas, onde o teor de $\mathrm{FeO}$ apresentou valores maiores que $30 \%$ na escória. As composições químicas dessas escórias estão na Tabela 2.

Tabela 2. Composição química das escórias da literatura (EL) e com alto teor de $\mathrm{FeO}(\mathrm{EF})$

\begin{tabular}{|c|c|c|c|c|c|c|c|c|}
\hline ESCÓRIAS & $\begin{array}{c}\mathrm{FeO} \\
\mathbf{( \% )}\end{array}$ & $\begin{array}{c}\mathrm{CaO} \\
\mathbf{( \% )}\end{array}$ & $\begin{array}{c}\mathbf{S i O}_{\mathbf{2}} \\
\mathbf{( \% )}\end{array}$ & $\begin{array}{c}\mathbf{M n O} \\
\mathbf{( \% )}\end{array}$ & $\begin{array}{c}\mathbf{P}_{\mathbf{2}} \mathbf{O}_{\mathbf{5}} \\
\mathbf{( \% )}\end{array}$ & $\begin{array}{c}\mathbf{A l}_{\mathbf{2}} \mathbf{O}_{\mathbf{3}} \\
\mathbf{( \% )}\end{array}$ & $\begin{array}{c}\mathbf{M g O} \\
\mathbf{( \% )}\end{array}$ & $\mathbf{T}\left({ }^{\circ} \mathbf{C}\right)$ \\
\hline $\mathrm{EL}$ & 16,00 & 52,19 & 13,05 & 5,74 & 2,11 & 1,71 & 9,26 & 1676 \\
\hline $\mathrm{EF}$ & 35,31 & 40,10 & 10,08 & 4,99 & 1,49 & 1,83 & 6,21 & 1687 \\
\hline
\end{tabular}

A escolha dessas escórias foi feita devido à grande diferença entre os teores de FeO e $\mathrm{CaO}$ existente entre elas. Assim, foi realizada uma análise da influência desses teores na desfosforação. As fases presentes na escória foram determinadas através de difração de Raios-X e Termodinâmica Computacional.

\subsection{Utilização da termodinâmica computacional}

Foram feitas simulações no programa Factsage 6.2 do aquecimento das escórias estudadas, de maneira a determinar as fases formadas, fração de líquidos e sólidos presentes, dentre outros. Também foram realizadas simulações do equilíbrio metal-

* Contribuição técnica ao 45 Seminário de Aciaria - Internacional, 25 a 28 de maio de 2014, Porto Alegre, RS, Brasil. 
escória, de forma a determinar o teor de fósforo de equilíbrio, além de dados como atividade da cal, porcentagem de fase liquida e sólida, etc. Para cálculo do rendimento foram utilizados os seguintes teores de fósforo do gusa e fósforo final, dados na Tabela 3.

Tabela 3. Composição química do aço utilizado para a simulação de equilíbrio e cálculo do rendimento da desfosforação

\begin{tabular}{|ccc|}
\hline ESCÓRIAS & $\begin{array}{c}\text { P gusa } \\
\text { (\%) }\end{array}$ & $\begin{array}{c}\mathbf{P} \text { final } \\
\mathbf{( \% )}\end{array}$ \\
\hline EUA & 0,097 & 0,017 \\
\hline EUD & 0,1 & 0,013 \\
\hline EF & 0,081 & 0,019 \\
\hline
\end{tabular}

A partir dos resultados encontrados foram feitas as considerações necessárias para determinar a influência das fases sólidas na desfosforação do aço.

Assim, as composições das escórias utilizadas neste trabalho foram listadas em ordem decrescente com relação ao teor de $\mathrm{CaO}$, na Tabela 4.

Tabela 4. Composição química das escórias utilizadas no presente trabalho e suas respectivas basicidades

\begin{tabular}{|ccccccccc|}
\hline EScóRIAS & $\begin{array}{c}\mathrm{FeO} \\
\mathbf{( \% )}\end{array}$ & $\begin{array}{c}\mathrm{CaO} \\
\mathbf{( \% )}\end{array}$ & $\begin{array}{c}\mathrm{SiO}_{2} \\
\mathbf{( \% )}\end{array}$ & $\begin{array}{c}\mathrm{MnO} \\
\mathbf{( \% )}\end{array}$ & $\begin{array}{c}\mathbf{P}_{2} \mathrm{O}_{5} \\
\mathbf{( \% )}\end{array}$ & $\begin{array}{c}\mathrm{Al}_{2} \mathrm{O}_{3} \\
\mathbf{( \% )}\end{array}$ & $\begin{array}{c}\mathrm{MgO} \\
\mathbf{( \% )}\end{array}$ & Basicidade \\
\hline $\begin{array}{c}\text { Escória da } \\
\text { literatura }\end{array}$ & 16,00 & 52,19 & 13,05 & 5,74 & 2,11 & 1,71 & 9,26 & 3,999 \\
\hline $\begin{array}{c}\text { Escória } \\
\text { Usiminas antes }\end{array}$ & 24,70 & 47,20 & 11,08 & 5,74 & 2,11 & 1,71 & 9,26 & 4,259 \\
\hline $\begin{array}{c}\text { Escória } \\
\text { Usiminas } \\
\text { depois }\end{array}$ & 24,80 & 45,30 & 11,17 & 5,74 & 2,59 & 1,71 & 9,26 & 4,056 \\
\hline $\begin{array}{c}\text { Escória } \\
\text { elaborada }\end{array}$ & 35,31 & 40,10 & 10,08 & 4,99 & 1,49 & 1,83 & 6,21 & 3,978 \\
\hline
\end{tabular}

Com os valores das composições químicas das escórias foram feitos cálculos dos coeficientes de atividade e partição do fósforo, basicidade baseado nas equações dos autores citados na introdução e os valores encontrados foram analisados e discutidos.

\section{RESULTADOS E DISCUSSÃO}

\subsection{Determinação das Fases}

O difratograma de raios-X das escórias estudadas pela USIMINAS é apresentado na Figura 2. As principais fases identificadas são: silicato dicálcio $\left(2 \mathrm{CaO} . \mathrm{SiO}_{2}\right)$, magnésio-wustita (MgO.FeO), cálcio-ferrita ( $\mathrm{CaO} . \mathrm{FeO})$, brownmillerita $\left(4 \mathrm{CaO} \cdot \mathrm{Al}_{2} \mathrm{O}_{3} \cdot \mathrm{FeO}\right)$, wustita $(\mathrm{FeO})$ e periclásio $(\mathrm{MgO})$. De acordo com a intensidade dos picos nos difratogramas, a fase principal é o silicato dicálcico, seguida em ordem pela magnésio-wustita e cálcio-ferrita [18].

\footnotetext{
* Contribuição técnica ao 450 Seminário de Aciaria - Internacional, 25 a 28 de maio de 2014, Porto Alegre, RS, Brasil.
} 

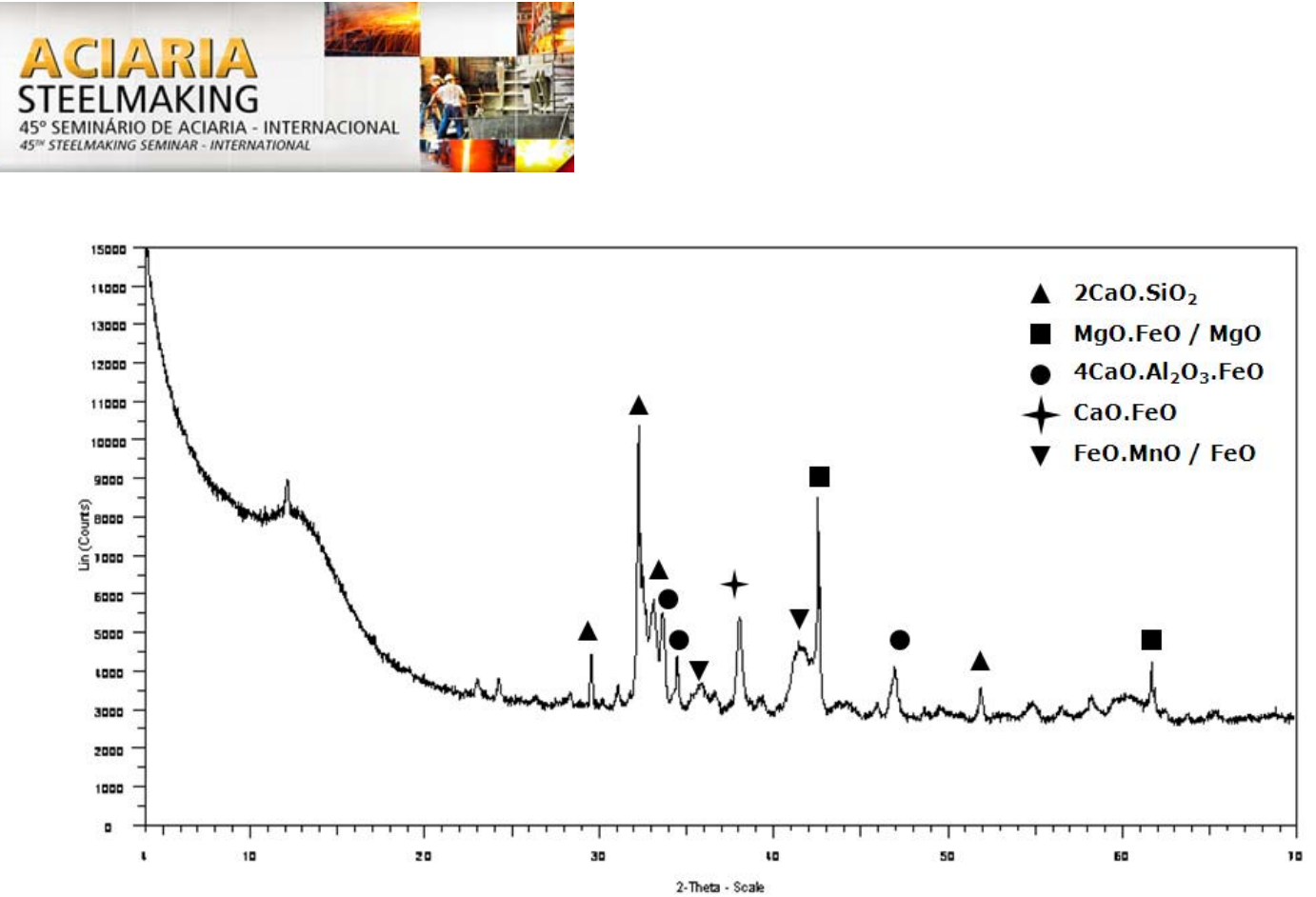

Figura 2. Difratometria de raios $X$ com identificação das principais fases da escória de convertedor LD [18].

Pelo difratograma é possível identificar a presença de precipitados de $\mathrm{CaO}$ e silicato dicálcico que estão sólidos na temperatura de fabricação do aço. Sendo assim, a sua presença aumenta a viscosidade da escória, prejudicando a interação do aço com a escória, reduzindo a eficiência de desfosforação.

A simulação do aquecimento das escórias pelo Factsage forneceu a composição química das escórias liquidas nas temperaturas de processo de cada uma, listada na Tabela 5.

Tabela 5. Composição química da escória líquida e sólida no aquecimento.

\begin{tabular}{|c|c|c|c|c|c|c|c|c|c|}
\hline ESCÓRIAS & $\begin{array}{l}\mathrm{FeO} \\
(\%)\end{array}$ & $\begin{array}{l}\mathrm{CaO} \\
(\%)\end{array}$ & $\begin{array}{c}\mathrm{SiO}_{2} \\
(\%)\end{array}$ & $\begin{array}{r}\text { MnO } \\
(\%)\end{array}$ & $\begin{array}{c}\mathrm{Ca}_{3}\left(\mathrm{PO}_{4}\right)_{2} \\
(\%)\end{array}$ & $\begin{array}{c}\mathrm{Al}_{2} \mathrm{O}_{3} \\
(\%)\end{array}$ & $\begin{array}{l}\text { MgO } \\
\text { (\%) }\end{array}$ & $\begin{array}{c}\text { CaO } \\
\text { sólido } \\
\text { (\%) }\end{array}$ & $\begin{array}{c}\text { MgO } \\
\text { sólido } \\
\text { (\%) }\end{array}$ \\
\hline EUA & 27,32 & 45,15 & 12,25 & 4,79 & 509 & 1,75 & 3,65 & 3,87 & 5,67 \\
\hline EUD & 26,74 & 44,39 & 12,06 & 6,20 & 4,98 & 1,85 & 7 & , & 5,75 \\
\hline EL & 18,74 & 47 & 15 & 6,70 & 5,3 & 2,00 & 4 & 9,32 & 5,35 \\
\hline EF & 35,88 & 38,95 & 10,24 & 5,07 & 3,31 & 1,86 & 4,69 & - & 1,58 \\
\hline
\end{tabular}

Analisando-se a Tabela 5 tem-se que em nenhuma das escórias estudadas houve a formação de silicato dicálcico líquido ou sólido. Além da composição química das escórias, também foi possível obter com o programa os seguintes gráficos de fases, representados na Figura 3.

\footnotetext{
* Contribuição técnica ao 45 Seminário de Aciaria - Internacional, 25 a 28 de maio de 2014 , Porto Alegre, RS, Brasil.
} 


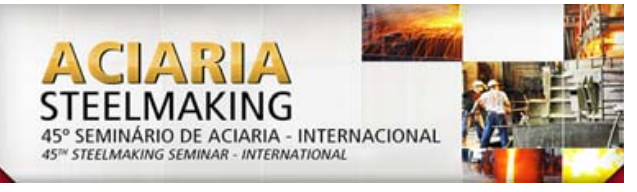

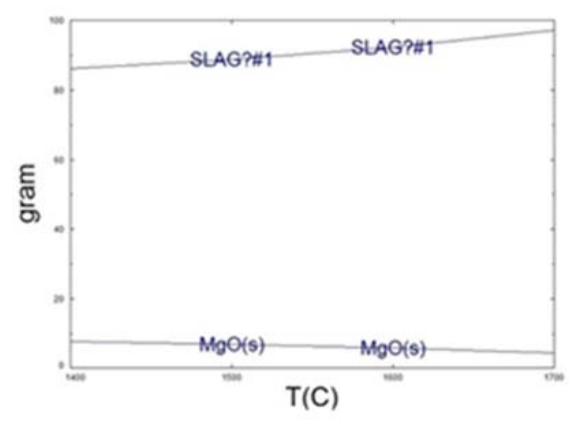

(a)

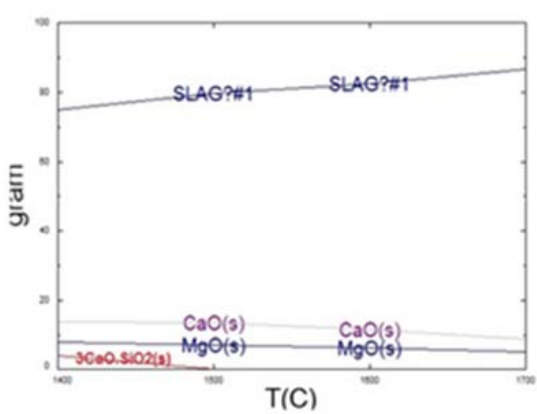

(c)

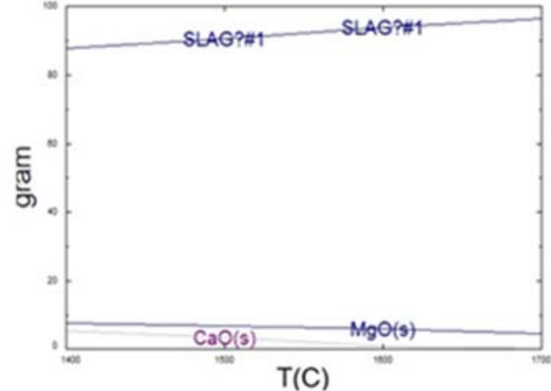

(b)

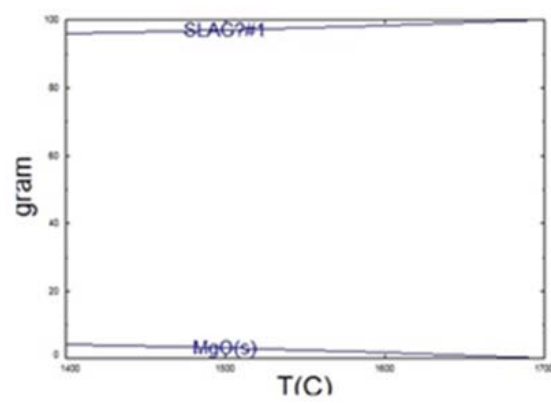

(d)

Figura 3. Gráficos das fases sólidas e liquidas das escórias estudadas ((a) escória USIMINAS antes, (b) escória Usiminas depois, (c) escória da literatura e (d) escória elaborada) na faixa de temperatura de $1400^{\circ} \mathrm{C}$ a $1700^{\circ} \mathrm{C}$. A fase SLAG\#1 representa todas as fases que se encontram líquidas.

Observando-se os gráficos tem-se que a fase de silicato dicálcico não se forma para as escórias estudadas nem no estado líquido nem sólido. A fase $3 \mathrm{CaO} \mathrm{SiO}_{2}$

formou-se sólida na escória da literatura em temperaturas abaixo da estudada (entre $1400^{\circ} \mathrm{C}$ e $1500^{\circ} \mathrm{C}$ ). Nas escórias da Usiminas, antes e depois, observa-se apenas a presença das fases sólidas $\mathrm{MgO}$ e $\mathrm{CaO}$ e na escória elaborada apenas $\mathrm{MgO}$, como mostrado na tabela 5. Assim, é possível concluir que todo o $\mathrm{P}_{2} \mathrm{O}_{5}$ foi fixado pela cal na forma de $\mathrm{Ca}_{3}\left(\mathrm{PO}_{4}\right)_{2}$ e se encontra liquido na escória. Além disto, pode-se observar pelas figuras que a EUD obteve menor teor de CaO sólido na escória. Infere-se que a presença da fase $2 \mathrm{CaO} . \mathrm{SiO}_{2}$ na difração e não na simulação se deve ao fato de o processo de amostragem e resfriamento das amostras ter causado uma queda na temperatura que foi suficiente para a formação desta fase.

\subsection{Determinação dos Lps, coeficiente de atividade do $\mathrm{P}_{2} \mathrm{O}_{5}$ e rendimento}

Os valores de Lps encontrados para cada escória estão listados na Tabela 6, calculou-se também o coeficiente de atividade do pentóxido de fósforo para cada escória. Além disso, o rendimento real da desfosforação também foi calculado, utilizando-se a seguinte equação.

$$
\% D e P=\frac{P g u s a-P f d s}{P g u s a}
$$

\footnotetext{
* Contribuição técnica ao 450 Seminário de Aciaria - Internacional, 25 a 28 de maio de 2014, Porto Alegre, RS, Brasil.
} 
Tabela 6. Valores de Lps, rendimento real da desfosforação e coeficiente de atividade do $\mathrm{P}_{2} \mathrm{O}_{5}$

\begin{tabular}{|c|c|c|c|c|c|c|c|}
\hline ESCóRIAS & $\begin{array}{c}\text { Lp } \\
\text { Suito }\end{array}$ & $\begin{array}{c}\text { Lp } \\
\text { Healy }\end{array}$ & $\begin{array}{c}\text { Lp } \\
\text { Turkdogan }\end{array}$ & Lp Basu & $\begin{array}{c}\text { Lp } \\
\text { Real }\end{array}$ & $\begin{array}{c}\text { Coef. } \\
\text { Atividade } \\
\mathbf{P}_{\mathbf{2}} \mathbf{O}_{\mathbf{5}}\end{array}$ & $\begin{array}{c}\text { Rendimento } \\
\mathbf{( \% )}\end{array}$ \\
\hline EUA & 543,764 & 825,132 & 334,138 & 273,322 & 67,15 & $3,88 \mathrm{E}-17$ & 82,50 \\
\hline EUD & 466,508 & 585,593 & 250,193 & 261,051 & 70,72 & $6,36 \mathrm{E}-17$ & 87,00 \\
\hline EL & 490,260 & 698,684 & 258,790 & 266,283 & $-^{*}$ & $1,72 \mathrm{E}-16$ & $-^{*}$ \\
\hline EF & 190,460 & 161,020 & 124,430 & 211,530 & 33,55 & $1,88 \mathrm{E}-16$ & 76,54 \\
\hline
\end{tabular}

*Na literatura não há dados suficientes para cálculo do Lp real.

Comparando-se as escórias EUA e EUD tem-se que os valores de Lp de equilíbrio para EUA foram maiores que os encontrados para EUD. Porém o Lp real e o rendimento da escória EUD foram maiores. Isso ocorre porque Lp de equilíbrio é na medida termodinâmica, medindo apenas a partição de fósforo no equilíbrio. Já Lp real e o rendimento medem os valores reais dos processos industriais. Estes valores dependem além dos parâmetros termodinâmicos, de parâmetros cinéticos, como mostrado na equação 9.

Portanto, não necessariamente uma escória que tenha maiores valores de Lp de equilíbrio terá os maiores valores de Lp real e de rendimento de desfosforação.

A influência da termodinâmica na velocidade da reação é dada pelo termo $\left\{\left[\% P_{i}\right]-\left[\% P_{e q}\right]\right\}$, que é força motriz da reação. Quanto melhores as condições termodinâmicas menores os valores de Peq, e maior será o valor da força motriz e consequentemente da velocidade da reação.

Como pode ser notado pela equação 9 , a velocidade de desfosforação é também influenciada pela constante de velocidade $\mathrm{k}$. Esta constante depende da \% de liquido e de sólido e da viscosidade da escória, assim como da agitação do processo. Estes parâmetros não são levados em conta no cálculo de Lp de equilíbrio, e para um melhor entendimento dos valores de Lp real e do rendimento é preciso conhecê-los.

\subsection{Determinação de dados termodinâmicos e cinéticos}

Os dados termodinâmicos e cinéticos que influenciam na desfosforação foram obtidos por meio do programa da termodinâmica computacional o Factsage 6.2 e estão na Tabela 7.

Tabela 7. Fatores termodinâmicos e cinéticos obtidos pelo Factsage

\begin{tabular}{|l|l|l|l|l|l|l|}
\hline ESCÓRIAS & $\mathbf{a C a O}$ & $\mathbf{a F e O}$ & $\mathbf{a C a}\left(\mathbf{P O}_{4}\right)_{\mathbf{2}}$ & $\mathbf{\% L i q u i d o}$ & $\begin{array}{l}\mathrm{CaO} \text { sólido } \\
\mathbf{( \% )}\end{array}$ & $\begin{array}{l}\text { MgO sólido } \\
\mathbf{( \% )}\end{array}$ \\
\hline EUA & 1 & 0,138 & 0,066 & 90,43 & 3,87 & 5,67 \\
\hline EUD & 1 & 0,132 & 0,070 & 92,63 & 1,68 & 5,75 \\
\hline EL & 1 & 0,085 & 0,044 & 85,38 & 9,32 & 5,35 \\
\hline EF & 0,60 & 0,220 & 0,069 & 98,42 & - & 1,58 \\
\hline
\end{tabular}

Tabela 7. Fatores termodinâmicos e cinéticos obtidos pelo Factsage (Cont.)

\begin{tabular}{|l|l|l|l|l|l|}
\hline ESCÓRIAS & \%Sólido & \%Peq & $\gamma \mathbf{P}_{\mathbf{2}} \mathbf{O}_{\mathbf{5}}$ & $\begin{array}{l}\text { Viscosidade } \\
\text { (poise) }\end{array}$ & Rendimento \\
\hline EUA & 9,57 & 0,0026 & $3,88 \mathrm{E}-17$ & 0,6995 & 82,65 \\
\hline EUD & 7,37 & 0,0028 & $6,36 \mathrm{E}-17$ & 0,6368 & 86,60 \\
\hline EL & 14,62 & 0,0034 & $1,72 \mathrm{E}-16$ & 1,0119 & - $^{*}$ \\
\hline EF & 1,58 & 0,0029 & $1,88 \mathrm{E}-16$ & 0,4045 & 76,54 \\
\hline
\end{tabular}

\footnotetext{
* Contribuição técnica ao 450 Seminário de Aciaria - Internacional, 25 a 28 de maio de 2014, Porto Alegre, RS, Brasil.
} 
A Tabela 7 fornece diversas informações a respeito do aquecimento das escórias e do equilíbrio metal-escória nas temperaturas de cada uma, simulados pela termodinâmica computacional.

Uma primeira análise pode ser feita com relação à quantidade de $\mathrm{CaO}$ sólido formado. A escória EL apresentou mais de $9 \%$ de $\mathrm{CaO}$ sólido. Além disso, esta escória apresentou a maior porcentagem de sólidos de todas as escórias. Isto é devido principalmente ao alto teor de $\mathrm{CaO}$. Este teor de $\mathrm{CaO}$ não é necessário, pois somente está contribuindo para aumentar a viscosidade da escória como mostrado na Tabela 7. Este aumento na viscosidade causa uma diminuição no valore de $k$. Porém esta escória tem a mesma atividade de $\mathrm{CaO}$ que as escórias EUA e EUD que é 1, e praticamente o mesmo teor de Peq. Esta escória provavelmente terá um menor Lp real e menor rendimento de desfosforação que as escórias EUA EUD em mesmas condições.

As escórias EUA e EUD apresentaram menores teores de CaO sólido, e mesmo após a diminuição da quantidade de $\mathrm{CaO}$, a escória continuou saturada, com atividade igual a 1, permitindo assim, uma diminuição da quantidade da cal utilizada durante o processo de refino primário. Além disso, as condições termodinâmicas foram mantidas e as cinéticas foram melhoradas.

A escória EF, apesar de uma maior quantidade de líquidos, teve o menor Lp real e o menor rendimento, devido à baixa atividade do $\mathrm{CaO}$, que é de 0,6.

Pode-se afirmar que, a melhor escória desfosforante é aquela que tiver a atividade do $\mathrm{CaO}$ igual a 1 e maior quantidade de liquido possível.

Observando-se a Tabela 7 é possível concluir que não há a formação do silicato dicálcico sólido, uma vez que os compostos que estão sólidos a essa temperatura são a cal e o óxido de magnésio. Isso pode ser explicado pela temperatura que foi analisada as amostras de escória da Usiminas no difratograma, uma vez que o silicato dicálcico sólido se forma durante a solidificação da escória, logo, em temperaturas elevadas, como a deste trabalho, esse composto não se forma nem sólido nem líquido. A presença de $\mathrm{CaO}$ sólido é importante pois, termodinamicamente, atividade da cal igual a 1 é um fator que melhora a eficiência da reação de desfosforação. Já a presença de $\mathrm{MgO}$ sólido possibilita um aumento da vida útil do refratário do convertedor.

Os fatores termodinâmicos relacionam-se com a atividade da cal, atividade do $\mathrm{FeO} e$ do fosfato tricálcio liquido formado, como pode ser observado pela equação 1.

Assim, maiores atividades da cal e menores atividades do $\mathrm{FeO}$ e do $\mathrm{Ca}_{3}\left(\mathrm{PO}_{4}\right)_{2}$, permitem a obtenção de menores teores de fósforo final, de acordo com a Equação 1.

\section{CONCLUSÃO}

Pelas condições do presente trabalho é possível concluir que:

- Os ajustes promovidos pela empresa Usiminas em suas escórias atingiram o objetivo visado da empresa;

- Não houve a formação do silicato dicálcio líquido ou sólido na temperatura analisada;

- Todo $\circ \mathrm{P}_{2} \mathrm{O}_{5}$ foi fixado pela cal e encontra-se na forma de fosfato tricálcico $\left(\mathrm{Ca}_{3}\left(\mathrm{PO}_{4}\right)_{2}\right)$ líquido na escória.

- As fases sólidas formadas neste trabalho, $\mathrm{CaO}$ e $\mathrm{MgO}$, influenciam positivamente o processo no convertedor, uma vez que, termodinamicamente, uma escória saturada em $\mathrm{CaO}$ promove uma maior desfosforação. Além

* Contribuição técnica ao 45 Seminário de Aciaria - Internacional, 25 a 28 de maio de 2014, Porto Alegre, RS, Brasil. 
disso, a saturação do $\mathrm{MgO}$ permite um aumento da vida útil do refratário do convertedor.

- Os valores de Lps, calculados baseados nas equações de diversos autores, mostraram que a melhor escória desfosforante é a escória EUA. Porém, esta escória não foi a mais eficiente. Portanto, a análise da eficiência de uma escória segundo os valores de Lp de equilíbrio não é eficiente;

- Maiores percentuais de sólidos influenciam na cinética da reação de desfosforação, pois prejudicam a interação metal-escória, uma vez que as reações no convertedor se processam na fase líquida. Assim, pode ser por esse motivo que a escória da literatura apresentou valores mais baixos de fósforo de equilíbrio.

- A melhor escória desfosforante é aquela que tiver a atividade do $\mathrm{CaO}$ igual a 1 e maior quantidade de líquido possível.

\section{Agradecimentos}

Os autores agradecem à CAPES pela bolsa de mestrado, à ABM pela oportunidade de apresentação deste trabalho no $45^{\circ}$ Seminário de Aciaria - Internacional e a Usiminas pela parceria neste trabalho.

\section{REFERÊNCIAS}

1 Turkdogan ET. Fundamentals of steelmaking. London: The Institute of Materials; 1996.

2 Turkdogan ET, Pearson J. Activity of constituents of iron and steelmaking slags. Journal of the Iron and Steel Institute. 1953:175:398.

3 Balajiva K, Quarrell AG, Vajragupta P. Journal of the Iron and Steel Institute. 1946;153:115.

4 Suito $\mathrm{H}$, Inoue R. Behavior of phosphorous transfer from $\mathrm{CaO}-\mathrm{Fe}_{t} \mathrm{O}-\mathrm{P}_{2} \mathrm{O}_{5}\left(-\mathrm{SiO}_{2}\right)$ slag to CaO particles. ISIJ International. 2006;46(2):180-187.

5 Healy G. A new look at phosphorus distribution. Journal of the Iron and Steel Institute. 1970;208(6): 664-668.

6 Basu S, Lahiri K, Seetharaman S. A model for activity coefficient of P2O5 in BOF slag and phosphorus distribution between liquid steel and slag. ISIJ International. 2007;47(8):1236-38.

7 Rizzo E. Introdução aos processos de refino primário dos aços nos convertedores a oxigênio. São Paulo: ABM - Associação Brasileira de Metalurgia e Materiais; 2005. p.109.

8 Ghosh A, Chtatterjee A. Ironmaking and steelmaking: theory and practice. $1^{\mathrm{a}}$ ed. New Delhi: PHI Learning Pvt. Ltd.; 2011. p.280.

9 Chang K, Huang C, Huang W, Liu Y. Investigations of microstructure and phosphorus distribution in BOF slag. Hsiao Kang: China Steel Technical Report No.21; 2008. p.1-6.

10 Monaco A, Lu W-K. The properties of steel slag aggregates and their dependence on melt shop practices. In: 55th Ironmaking Conference Proceedings; 1996; Pittsburgh, USA. 1996. p.705-715

11 Tossavainen M, Engstrom F, Yang Q, Menad N, Lidstrom M, Bjorkman B. Characteristics of steel slag under different cooling conditions. Review Waste Management. 2007;27:1335-44.

12 Inoue $\mathrm{R}$, Suito $\mathrm{H}$. Phosphorus partition between $2 \mathrm{CaO} . \mathrm{SiO}_{2}$ particles and $\mathrm{CaO}-$ $\mathrm{SiO}_{2}-\mathrm{FeO}$ slags. ISIJ International. 2006;46(2):174-79.

* Contribuição técnica ao 45 Seminário de Aciaria - Internacional, 25 a 28 de maio de 2014, Porto Alegre, RS, Brasil. 
13 Basu S, Lahiri A-K, Seetharaman S. Distribution of phosphorus and oxygen between liquid steel and basic oxygen steelmaking slag. La Revue de Métallurgie - CIT. Paris; 2009. p.21-26.

14 Campos VF. Tecnologia de fabricação do aço líquido. $3^{a}$ ed. Belo Horizonte: Universidade Federal de Minas Gerais; 1985. p.259.

15 Wei P, Sano M, Hirasawa M, Mori K. Kinects of phosphorus transfer between iron oxide containing slag and molten iron of high carbon concentration under $\mathrm{Ar}-\mathrm{O}_{2}$ atmosphere. ISIJ International. 1993;33(4):479-87.

16 Costa e Silva ALV. Cálculos de equilíbrio em aciaria através da termodinâmica computacional. Tecnologia em Metalurgia e Materiais. 2006;3(1):45-52.

17 Gaye H. Computational thermodynamics as a tool for clean steel making: some new developments and a few older ones. In: $7^{\text {th }}$ International Conference on Clean Steel; 2007; Balatonfüred, Hungary. 2007.

18 Parreiras HF, Carneiro CAR, Araujo TCS. Estudo microestrutural da escória de convertedor LD e sua relação com o processo de desfosforação. In: ABM. 44 ${ }^{\circ}$ Seminário de Aciaria - Internacional; 26-29 mai. 2013; Araxá, Brasil. São Paulo: ABM; 2013.

* Contribuição técnica ao 45 Seminário de Aciaria - Internacional, 25 a 28 de maio de 2014, Porto Alegre, RS, Brasil. 